\title{
Shared Aperture Multibeam Forming of Time-Modulated Linear Array
}

\author{
Li-juan Sun, ${ }^{1}$ Zhen-kai Zhang $\mathbb{D}^{1,2}$ and Hamid Esmaeili Najafabadi ${ }^{2}$ \\ ${ }^{1}$ College of Electronics Information, Jiangsu University of Science and Technology, Zhenjiang 212003, China \\ ${ }^{2}$ Department of Electrical and Computer Engineering, University of Calgary, Calgary ABT2N1N4, Canada
}

Correspondence should be addressed to Zhen-kai Zhang; zhangzhenkai@just.edu.cn

Received 18 May 2019; Revised 28 July 2019; Accepted 13 August 2019; Published 26 November 2019

Academic Editor: Michele Scarpiniti

Copyright (c) 2019 Li-juan Sun et al. This is an open access article distributed under the Creative Commons Attribution License, which permits unrestricted use, distribution, and reproduction in any medium, provided the original work is properly cited.

A novel technique is proposed in this paper for shared aperture multibeam forming in a complex time-modulated linear array. First, a uniform line array is interleaved randomly to form two sparse array subarrays. Subsequently, the theory of time modulation for linear arrays is applied in the constructed subarrays. In the meantime, the switch-on time sequences for each element of the two subarrays are optimized by an optimized differential evolution (DE) algorithm, i.e., the scaling factor of the sinusoidal iterative chaotic system and the adaptive crossover probability factor are used to enhance the diversity of the population. Lastly, the feasibility of the new technique is verified by the comparison between this technique and the basic multibeam algorithm in a shared aperture and the algorithm of iterative FFT. The results of simulations confirm that the proposed algorithm can form more desired beams, and it is superior to other similar approaches.

\section{Introduction}

In recent years, electromagnetic environments have been more complicated due to the increased number and complexity of antennas, and they have been vital to many fields $[1,2]$. Shared aperture technology is one of the feasible approaches to achieve multifunction application of the array antenna $[3,4]$. This technology enables two or more antenna subarrays to occupy one aperture by space-division multiplexing, in which each subarray can perform different functions. This method can not only reduce the number of antennas, but also effectively avoid electromagnetic compatibility problems between the antenna units. Recently, some promising results in sharing aperture antennas have been achieved. In [5-8], the differential set theory was applied to implement the sparsely interlaced array of multisubarrays, in which the pattern of each subarray is quite different and requires further optimization. In [9-11], using the Fourier transform between the array element excitation and the pattern, a multi-subarray interleaved shared aperture design method was proposed based on spectral energy matching of the subarray antenna pattern, in which the energy of the antenna pattern distributes to each subarray evenly. However, the yielded subarray pattern exhibits a higher peak sidelobe level (PSL), so it cannot flexibly control beam pointing. The literature $[12,13]$ discussed the method to reduce the PSL in linear antenna arrays by genetic algorithms (GA), whereas it cannot be applicable to the shared aperture multibeam forming. The literature [14-17] also employed intelligent algorithms to optimize the location of array elements. Though the above-shared aperture methods can implement sparsely interleaved multi-subarray, there are also unsolved problems (e.g., the large difference in the pattern of each subarray, high PSL, and inflexible beam steering).

In this paper, a new shared aperture method is proposed based on the time-modulated linear array (TMLA) for shared aperture multibeam forming. TMLA has been adopted as a means of synthesizing low/ultralow sidelobe patterns exhibiting a simple system structure and low cost. At present, the research based on TMLA is primarily about sideband suppression, pattern synthesis, and harmonic beamforming compared with conventional antenna arrays. The TMLA introduces a time variable into the conventional array antenna to control its performance. Each element is 
connected to a Radio Frequency (RF) switch. The excitation of the antenna array element and aperture excitation distribution is controlled by controlling the working state of the RF switch in the access array to achieve harmonic beamforming. The amplitude of each harmonic component is consistent with the harmonic distribution of $\sin (\cdot)$ function, and the magnitudes of low-order harmonic components are relatively large. Accordingly, it is more suitable to select only low-order harmonic components for beamforming. In this study, the first harmonic is selected for beamforming. Traditional shared aperture can only create two beams while forming two subarrays. In contrast, the shared aperture based on the TMLA can create five different desired beams using the random sparse interleaving. By optimizing the control time sequence by an optimized differential evolution algorithm, the PSL of each beam can be further reduced. In recent years, numerous studies have been published on the pattern synthesis of time-modulated array antennas. The literature [18-20] studied the synthesis of the TMLA patterns. The synthesis of simultaneous and multiple harmonic beams for applications (e.g., beam steering) has been already investigated $[21,22]$ to enhance the reliability of the communication system. The literature [23-26] presented pattern synthesis and steerable energy patterns in a two-dimensional planar time modulation array. Besides, in recent works, novel timed arrays of linear polarised Vivaldi antennas have been proposed [27]. Novel techniques for circularly polarised UWB-scanned patterns were explored with low sidelobes in [28]. Unlike [18-26], the present study can create beams at more than two different desired directions. Thus, the TMLA is adopted to solve the shared aperture multibeam forming problems for the first time.

The rest of this paper is organized as follows. In Section 2, the mathematical model of the time-modulated linear array is presented. In Section 3, the theory of the novel shared aperture multibeam forming technique is explained, and the optimized DE algorithm is presented. In Section 4, simulations are performed to assess the performance of the proposed method. Lastly, the conclusion is drawn in Section 5.

\section{Mathematical Model of Time-Modulated Linear Array}

A uniform linear array (ULA) with $N$ elements is considered, in which the element spacing $d$ is $1 / 2$ wavelength, and the array elements are ideal omnidirectional units. The radiation pattern is expressed as

$$
F(\theta)=\sum_{n=0}^{N-1} A_{n} e^{j n \beta d \sin \theta}
$$

where $A_{n}$ denotes the static excitation amplitude of the $n$-th element; $\beta=2 \pi / \lambda$ the wavenumber; $d$ the element spacing; and $\theta$ the beam scanning angle. According to TMLA, when all $N$ elements are time modulated, equation (1) can be rewritten as

$$
F(\theta)=\sum_{n=0}^{N-1} A_{n} U_{n}(t) e^{j n \beta d \sin \theta}
$$

where $U_{n}(t)$ denotes the periodic signal of the $n$-th element. The structure of time-modulated antenna array is shown in Figure 1.

In this paper, the array is controlled by the RF switch connected to each array element. The waveform of the periodic control timing of the $n$-th array element is shown in Figure 2, and its mathematical expression is

$$
U_{n}(t)= \begin{cases}1, & t_{\text {on }} \leq t \leq t_{\text {on }}+t_{d} \\ 0, & \text { otherwise }\end{cases}
$$

where $t_{\text {on }}$ denotes the instant time of the switch opening and $t_{d}$ the duration of the switch closure time.

The $U_{n}(t)$ denotes a gate function, which can be expanded to a Fourier series according to the nature of the periodic functions as expressed below:

$$
U_{n}(t)=\sum_{k=-\infty}^{\infty} c_{n k} e^{j 2 \pi k f_{m} t}
$$

where $T_{m}$ denotes the time modulation period; $f_{m}=1 / T_{m}$ modulation frequency; and $c_{n k}$ the complex Fourier coefficient of $k$-th harmonic, which is expressed as

$$
c_{n k}= \begin{cases}\frac{\sin \left(\pi k f_{m} t_{d}\right)}{\pi k} e^{-j k \pi f_{m}\left(2 t_{\mathrm{on}, n}+t_{d, n}\right)}, & k \neq 0, \\ f_{m} t_{d}, & k=0 .\end{cases}
$$

Equation (5) suggests that the phase is always zero when $k=0$, i.e., the fundamental component always points to $0^{\circ}$. When $k \neq 0$, Fourier coefficients of the positive $k$-th harmonic component and the negative $k$-th harmonic component are symmetric with respect to the array axis. Accordingly, the pattern of the TMLA is expressed as

$$
F_{k}(\theta)=\sum_{n=0}^{N-1} A_{n} \sin \left(\pi k f_{m} t_{d}\right) e^{-j k \pi\left(2 t_{\mathrm{on}, n}+t_{d, n}\right)} e^{j n \beta d \sin \theta} .
$$

Equation (6) shows that the $k$-th harmonic component generated by TMLA is a complex number. The excitation of the harmonic component is associated with the duration of the opening of the RF switch and the harmonic order $k$, while its phase is only associated with the central moment of the opening and closing times. Thus, when beamforming is performed by TMLA, it is appropriate to select lower-order harmonic components. The positive and negative first harmonics are taken only to point to the desired direction. Thus, the fundamental and positive and negative first harmonic components of the pattern are calculated as follows:

$$
\begin{aligned}
F_{0}(\theta) & =\sum_{n=0}^{N-1} A_{n} T_{\mathrm{dn}} e^{j n \beta d \sin \theta}, \\
F_{1}(\theta) & =\sum_{n=0}^{N-1} A_{n} \sin \left(\pi T_{\mathrm{dn}}\right) \times e^{-j \pi\left(2 T_{\mathrm{sn}}+T_{\mathrm{dn}}\right)} \times e^{j n \beta d \sin \theta}, \\
F_{-1}(\theta) & =\sum_{n=0}^{N-1} A_{n} \sin \left(\pi T_{\mathrm{dn}}\right) \times e^{j \pi\left(2 T_{\mathrm{sn}}+T_{\mathrm{dn}}\right)} \times e^{j n \beta d \sin \theta},
\end{aligned}
$$

where $T_{\mathrm{sn}}=t_{\mathrm{on}, n} / T_{m}, T_{\mathrm{dn}}=t_{d, n} / T_{m}$. By scrutinizing (7)-(9), it can be observed that since the range of the $\sin (x)$ function 


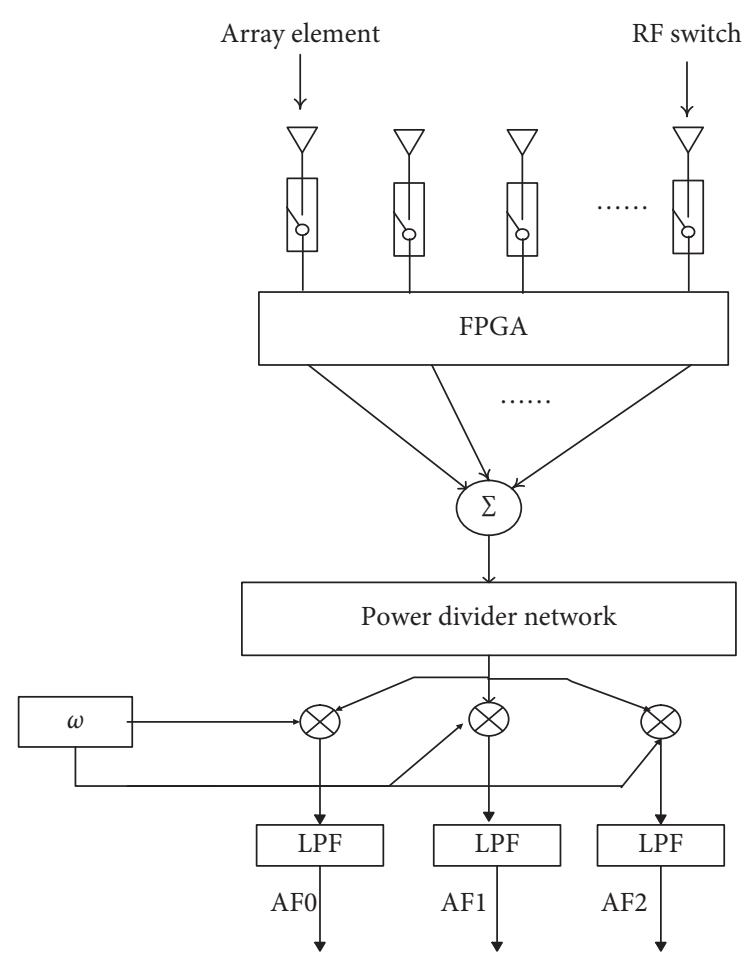

Figure 1: Basic structure for time modulation array.

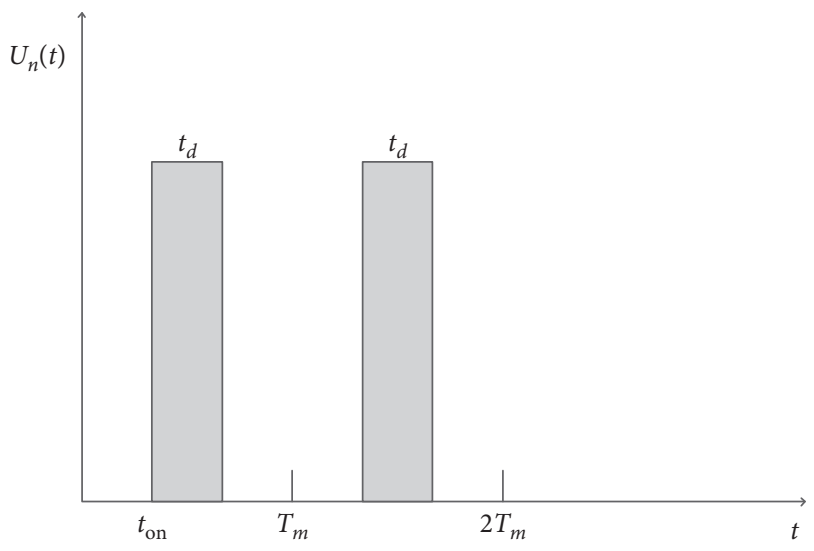

FIgURE 2: Control timing of the $n$-th array element.

is $[0,1]$, the range of $\sin \left(\pi T_{\mathrm{dn}}\right)$ is also $[0,1]$, suggesting that $T_{\mathrm{dn}}$ varies in $[0,0.5]$. To make the beam phase shift vary in $[-\pi, \pi], T_{\mathrm{dn}}$ should adopt a value in $[-0.5,0.5]$. For the first harmonic, $G_{n}=A_{n} c_{n 1}=A_{n} \sin \left(\pi T_{\mathrm{dn}}\right)$, where $G_{n}$ denotes the amplitude of the beam pattern, and it is assumed that $G_{n}$ is a given random sequence in $[0,1]$. Besides, denote the direction of the first harmonic component by $\theta_{0}$. Subsequently, the control sequence is expressed as

$$
\begin{aligned}
& T_{\mathrm{dn}}=\frac{1}{\pi} \cdot \arcsin \left(\frac{G_{n}}{A_{n}}\right), \\
& T_{\mathrm{sn}}=\frac{1}{2}\left[\frac{\left(n \beta d \sin \theta_{0}\right)}{\pi}-T_{\mathrm{dn}}\right] .
\end{aligned}
$$

From (6), it can be observed that the excitation and phase synthesis of the harmonic components can be obtained by adjusting the control sequences. Accordingly, the harmonic excitation of the TMLA can be utilized for the beamforming. The proposed TMLA-based shared aperture technique is based on sparse interleaving to yield two subarrays. By controlling the control sequences of the two subarrays, each subarray can yield beams with different directions.

In the meantime, the PSL of the antenna pattern is expressed as

$$
\left\{\begin{array}{l}
\mathrm{PSL}_{0}=\max \left(\left|F_{0}(\theta)\right|_{\theta \in S_{0}}\right), \\
\mathrm{PSL}_{1}=\max \left(\left|F_{1}(\theta)\right|_{\theta \in S_{1}}\right), \\
\mathrm{PSL}_{-1}=\max \left(\left|F_{-1}(\theta)\right|_{\theta \in S_{-1}}\right), \\
\mathrm{PSL}_{2}=\max \left(\left|F_{2}(\theta)\right|_{\theta \in S_{2}}\right),
\end{array}\right.
$$

where $F_{0}(\theta), F_{1}(\theta), F_{-1}(\theta), F_{2}(\theta)$, and $F_{-2}(\theta)$ denote the fundamental wave of the subarray 1 , the positive first-order harmonics of the subarray 1 , the negative first-order harmonics of the subarray 1 , the positive first-order harmonics of the subarray 2, and the negative first-order harmonics of the subarray 2 , respectively. Besides, $S_{0}, S_{1}, S_{-1}, S_{2}$, and $S_{-2}$ refer to the sidelobe regions of the corresponding beams, respectively. The following objective function is proposed as

$$
f(x)=\alpha\left(\mathrm{PSL}_{0}-\mathrm{SLL}\right)+\beta\left(\mathrm{PSL}_{1}-\mathrm{SLL}\right)+\gamma\left(\mathrm{PSL}_{2}-\mathrm{SLL}\right),
$$

where $\mathrm{PSL}_{0}, \mathrm{PSL}_{1}$, and $\mathrm{PSL}_{2}$ denote the PSL values for the fundamental, subarray 1 , and subarray 2 , respectively. SLL represents the desired value for the PSL. Also, $\alpha, \beta$, and $\gamma$ are the corresponding weight coefficient values. The multibeam PSL formed by this method is higher and will further be optimized by the optimized differential evolution (DE) algorithm.

\section{Shared Aperture Multibeam Forming Optimization}

In this section, the theory of the novel shared aperture multibeam forming technique is presented, and the DE algorithm is optimized here.

\subsection{Differential Evolution Algorithm}

3.1.1. Standard Differential Evolution Algorithm. DE refers to a population-based global optimization algorithm. It adopts a random search strategy and uses differential information between different individuals to guide the evolution of the entire population. Besides, it employs the greedy selection mechanism to control the evolution direction and search for the optimal solution that satisfies the conditions. The advantage of the DE algorithm is that its control parameters are relatively small, simple to implement, reliable, and fast $[29,30]$. The use of differential information of individual populations to achieve variation exhibits better stability. In the generation process of the offspring, the 
greedy competition mechanism is adopted to ensure the evolution of the population [31-34]. The DE method is selected as the optimization algorithm for its advantage of simplicity and efficiency, as it can exploit the greedy selection mechanism to control the evolution direction and search for the optimal solution that satisfies the conditions. Furthermore, this method can be optimized straightforwardly. Without the loss of generality, the solution for a given $D$ dimensional continuous optimization problem is as follows:

$$
\begin{array}{ll}
\min & f(x) \\
\text { s.t. } & x \in S \subset R^{D} \text {, }
\end{array}
$$

where $D$ denotes the individual dimension, i.e., the number of elements and $S$ is the search domain. The algorithm flow is presented as follows:

(1) Set parameters, which include the population size $N_{\mathrm{P}}$, the maximum iteration number $G_{M}$ and the mutation factor $F$, and the hybridization probability $\mathrm{CR}$; an initial population is yielded, and the running algebra is set as $G=0$.

(2) The element excitation amplitude is initialized to a random number within $0-1$, and the control sequences $T_{\mathrm{dn}}$ and $T_{\mathrm{sn}}$ are calculated by equations (10) and (11). Set the duration $T_{\mathrm{dn}}$ as the optimization variable; the initial population is given by

$$
X^{G}=\left(x_{1}^{G}, x_{2}^{G}, x_{3}^{G}, \ldots, x_{N_{P}}^{G}\right)
$$

where $G$ denotes the number of iterations; the variation range of $X$ is controlled at $0-0.5$; the $i$-th vector in the group is

$$
x_{i}^{G}=\left[x_{i, 1}^{G}, x_{i, 2}^{G}, x_{i, 3}^{G}, \ldots, x_{i, D}^{G}\right] \text {. }
$$

To make the initial population cover the entire search space $R^{D}$, it is initialized as

$$
x_{i, j}^{0}=x_{\min j}+\operatorname{rand}(0,1)\left(x_{\max j}-x_{\min j}\right) \text {, }
$$

where $x_{\min j}$ and $x_{\max j}$ denote the extrema of the optimization variable. Also, $\operatorname{rand}(0,1)$ denotes a random number in $[0,1]$.

(3) Two individuals different from the individual are randomly selected in the initial population; the difference vector is multiplied by the mutation operator $F$ and then added to the mutant individual:

$$
x_{v, i}^{G}=x_{r 1}^{G}+F\left(x_{r 2}^{G}-x_{r 3}^{G}\right),
$$

where $r_{1}, r_{2}$, and $r_{3}$ represent three different numbers in $[1, N p]$.

(4) The cross operation of the vector after the mutation and the parent vector can enhance the diversity of the population. Common cross equations are presented as follows:

$$
x_{u, i}^{G+1}= \begin{cases}x_{v, i}^{G}, & \text { rand } \leq \mathrm{CR}, \\ x_{i}^{G}, & \text { otherwise }\end{cases}
$$

where rand is a random number.

(5) The selection operation follows the "greedy" search strategy and adopts individuals with small adaptation values as descendants, which is expressed as

$$
x_{i}^{G+1}= \begin{cases}x_{u, i}^{G}, & f\left(x_{u, i}^{G}\right) \leq f\left(x_{i}^{G}\right), \\ x_{i}^{G}, & \text { otherwise, }\end{cases}
$$

where $f(x)$ denotes the objective function, which can be calculated by the above equations (12) and (13). Lastly, the above operations are repeated until the best solution is selected.

3.1.2. Optimized Differential Evolution Algorithm. The DE algorithm has downsides, e.g., slow convergence rate and easy to fall into local optimum. Especially in the late stage of its evolution, the population diversity and the convergence speed decrease, which leads to the algorithm falling into local optimum [35]. In this study, an optimized DE algorithm is proposed, exploiting the scaling factor of the sinusoidal iterative chaotic system and the adaptive crossover probability factor (CR) to enhance the diversity of the population. The sinusoidal chaotic random sequence [36] refers to a variable that fluctuates within the interval $[0,1]$. It exhibits randomness, ergodicity, aperiodicity, and extreme sensitivity to initial values, making it suitable for generating nonrepeating pseudorandom numbers in the range $[0,1]$. When the chaotic random sequence is employed for the population initialization and optimization search, the algorithm is capable of verifiably avoiding from falling into the optimal local solution.

The scaling factor based on a sinusoidal iterative chaotic system is given by

$$
F(n)=\sin ^{2}(c \arcsin \sqrt{F(n-1)}),
$$

where $c$ denotes the weight coefficient, generally chosen from the interval $[0,3]$; also, the initial value of the mutation operator is chosen randomly, i.e., $F(0) \in \operatorname{rand}(0,1)$.

The adaptive $\mathrm{CR}$ is expressed as

$$
\mathrm{CR}_{G}=\mathrm{CR}_{l}+\left(C_{h}-\mathrm{CR}_{l}\right)\left(\frac{G_{m}-G}{G_{m}}\right),
$$

where $\mathrm{CR}_{h}$ and $\mathrm{CR}_{l}$ denote the maximum and minimum values of $\mathrm{CR}$, respectively. After numerous experiments, $\mathrm{CR}_{l}=0.1$ and $\mathrm{CR}_{h}=0.9$ are applicable to most optimization problems. fun $\max _{\max }$ and fun $_{\min }$ are the maximum and minimum values of the objective function, respectively. $G_{m}$ indicates the maximum number of iterations, and $G$ the current number of iterations. The optimized DE algorithm flow chart is shown in Figure 3.

3.2. Shared Aperture Multibeam Forming Design Algorithm. A shared aperture sparsely interleaved array is capable of reducing the number of antennas, saving space, and reducing load and manufacturing costs. In this study, the TMLA is employed to form more beams from multiple subarrays based on the interleaved shared aperture. The 


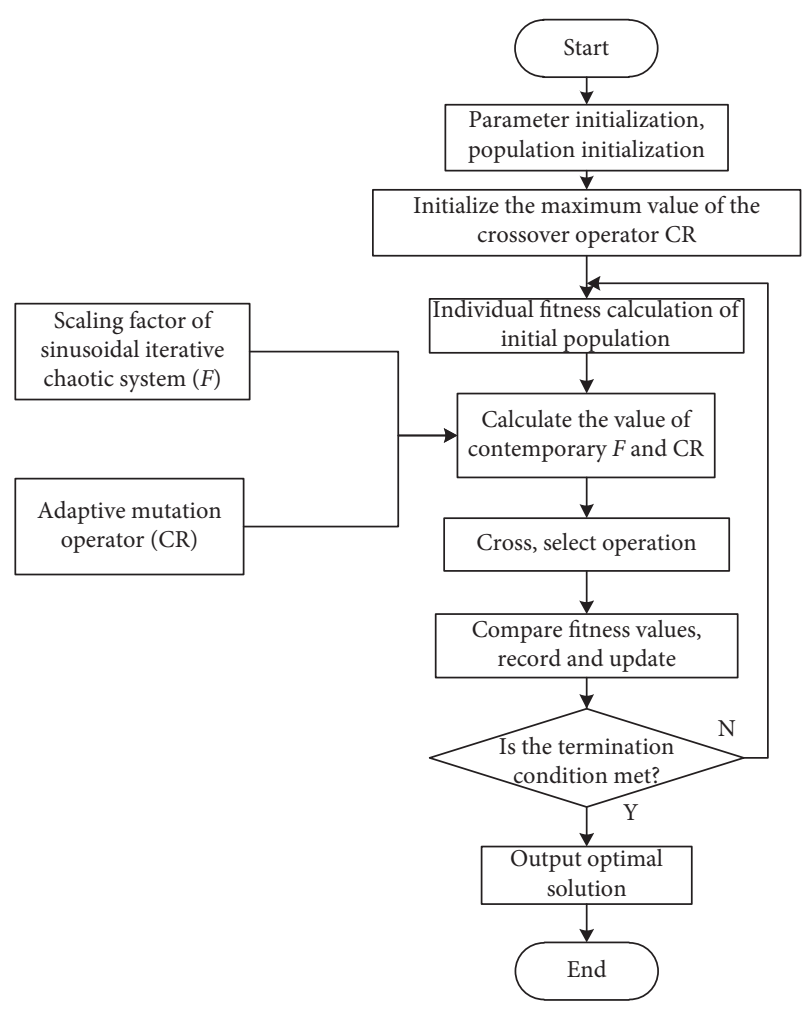

FIgURE 3: The optimized DE algorithm flow chart.

combination of shared aperture technology and the TMLA can yield more beams based on the same antenna resources and enhance the efficiency of the antenna utilization. The following is the locational distribution of the array elements with sparsely interleaved apertures, which is expressed as

Subarray 1: 1001100010110101101001010101100101010 10110100110000101010110

Subarray 2: $0110011101001010010110101010011010101-$ 01001011001111010101001

where 1 denotes an array of elements, 0 means free here. A uniform linear array is considered with $N$ elements. $N$ elements are chosen randomly from 0 to 1 , where 0 denotes the elements of subarray 1 , and 1 the array elements of subarray 2. After the two subarrays are formed by random sparse interleaving, the excitations for the two subarrays are initialized to the number $G_{n}$ in $[0,1]$ randomly. The proposed algorithm for multiple beamforming is presented in Algorithm 1.

\subsection{Multibeam Forming Based on Iterative FFT Algorithm.}

The iterative FFT algorithm exploiting the feature of the relationship between the uniform linear array element excitation and the Fourier transform converts the problem to determine the location of the subarray element into the problem to select the excitation energy of the array element. This method, based on the principle of density-weighted matrix and cross-selection, ascertain the location of the sparse subarray unit and achieve the uniform distribution of spectral energy of the subarray. In such a way, multibeam forming based on sparse interlaced arrays can be achieved.
Considering a linear array as shown in equation (1), the discrete inverse Fourier transform (DIFT) can be expressed as

$$
f(t)=\frac{1}{M} \sum_{n=0}^{M-1} F(n) e^{j n t \omega_{0}},
$$

where $M$ denotes the period and $\omega_{0}=2 \pi / M$ is the sampling interval. It can be observed from equations (1) and (23) that there is a Fourier transform relationship between the excitation $A$ of the array element and the array factor $F$.

Since there exists a Fourier transform relationship between the excitation and the pattern of the array antenna element, the fast Fourier transform (FFT) method can be adopted to convert the unit excitation and the pattern, significantly reducing the calculation amount and saving computing time. Taking the antenna design with $T$ subarrays interlaced as an example, the specific steps are presented as follows:

(1) Set the array excitation value $I$ to 1 according to a uniform linear array with sparse rate $R=1 / T$ random sparse array elements $N$.

(2) Perform an inverse FFT of $Q$ point on $I$ to calculate $P$.

(3) Determine the value of the sidelobe region of $P$, so the point value on the region larger than the constraint sidelobe value equals to the constraint sidelobe value, and the values at other points remain unchanged.

(4) Perform an FFT conversion of the $Q$ point on the corrected $P$ to yield a new excitation value $I$.

(5) Intercept the first $N$ values in $I$, yield a new excitation matrix, arrange them from large to small, and yield a new excitation vector.

(6) The excitation at the location $\left(I_{r}(i), I_{r}(i+T), \ldots\right.$, $\left.I_{r}(+T \times K)\right)$ corresponds to the location on $I$ as the location where the $i$-th subarray unit is located, and set the excitation value at the location as 1 .

(7) Perform an inverse FFT on the excitation vector of the new subarray 1 , determine whether the edge lobe peak of the updated antenna pattern changes with respect to the sidelobe peak at the previous iteration, and then proceeds to the next calculation; otherwise, output the result.

(8) The excitation sequence of the subarray 1 is entered as the new input value into the next iteration. Steps (2) to (8) are repeated until the newly generated antenna pattern sidelobe peaks no longer change, and output the optimized result.

\section{Experimental Simulation and Analysis}

The number of elements of the antenna array is set to 60 , the desired direction is $\theta_{1}= \pm 10^{\circ}$ and $\theta_{2}= \pm 30^{\circ}$. Referring to the literature $[37,38]$, and after simulating repeatedly, it is found that the beam pointing is only associated with the opening time $T_{\mathrm{sn}}$. The desired direction in this study is 
Input: The array number $N$, the number of samples $L$, target directions, the static excitation amplitude $A_{k}$, the parameters of the optimized DE algorithm $N_{p}, G_{m}, \mathrm{CR}$, and $F$.

Initialization: Randomly and sparsely interlace the line arrays of the $N$ array elements into two subarrays, and initialize the excitation of the two subarrays.

Calculate $F_{0}(\theta), F_{1}(\theta), F_{-1}(\theta), F_{2}(\theta)$, and $F_{-2}(\theta)$ using (7)-(9). Then $T_{\text {sn }}$ and $T_{\mathrm{dn}}$ can be calculated by (10) and (11). Lastly, the objective function value can be obtained by (11)-(13).

Iteration: While $G<G_{m}$

Adaptively updating the value of CR and $F$ using (21) and (22)

Generating variant individuals

Cross operation

Select the mutated individuals based on the objective function

If $f\left(x_{G_{-} \text {next }}\right)<f\left(x_{G}\right)$

record the optimal value of $f$ and $x_{G_{-} \text {next }}$, then update end loop.

End for

Output: Final weight vector $x_{1}, x_{2}$ and $f$.

Algorithm 1: Shared aperture multibeam forming for time-modulated arrays based on optimized DE algorithm.

stable, so the decision variable is only $T_{\mathrm{dn}}$. The specific parameter settings to optimize the DE algorithm are listed in Table 1.

4.1. Simulation Experiment 1. In TMLA harmonic beamforming, the first two harmonic components are larger, while the other subharmonic components are smaller. In this study, only the first harmonic component is applied for multibeam forming. The second harmonic is primarily considered suppressed as much as possible to reduce power loss. Take subarray 1 as an example. The fundamental wave, first harmonic, and second harmonic component formed by the underlying algorithm are presented in Figure 4. The patterns of the fully populated array and after optimization by the proposed algorithm are presented in Figures 5 and 6 . Also, the gain differences between the second harmonic component and the first harmonic component are shown in Table 2. The second harmonic component and the first harmonic component have a gain difference of $6.03 \mathrm{~dB}$ in the underlying algorithm. In the fully populated array, the gain difference between the second and first harmonic components is $6.36 \mathrm{~dB}$. After optimization by the proposed algorithm, the gain difference between the second and first harmonic components is increased to $18.03 \mathrm{~dB}$. Thus, the proposed algorithm significantly suppresses the second harmonic.

To verify the feasibility of the proposed algorithm, the algorithm of this study is simulated and compared with the beam performance formed by the basic TMLA shared aperture. The fundamental wave is set to point to $0^{\circ}$, while the positive first harmonic of subarray 1 points to $10^{\circ}$, and the positive first harmonic of subarray 2 points to $30^{\circ}$. The simulation results are presented in Figures 7-9.

The simulation diagram reveals that the PSL of the basic TMLA shared aperture multibeam forming fundamental wave is $-10.18 \mathrm{~dB}$, while the PSL of the positive first harmonic of the subarray 1 is $-10.01 \mathrm{~dB}$, and the PSL of the positive first harmonic of subarray 2 reaches $-11.31 \mathrm{~dB}$. After using the proposed optimized DE algorithm, the beam

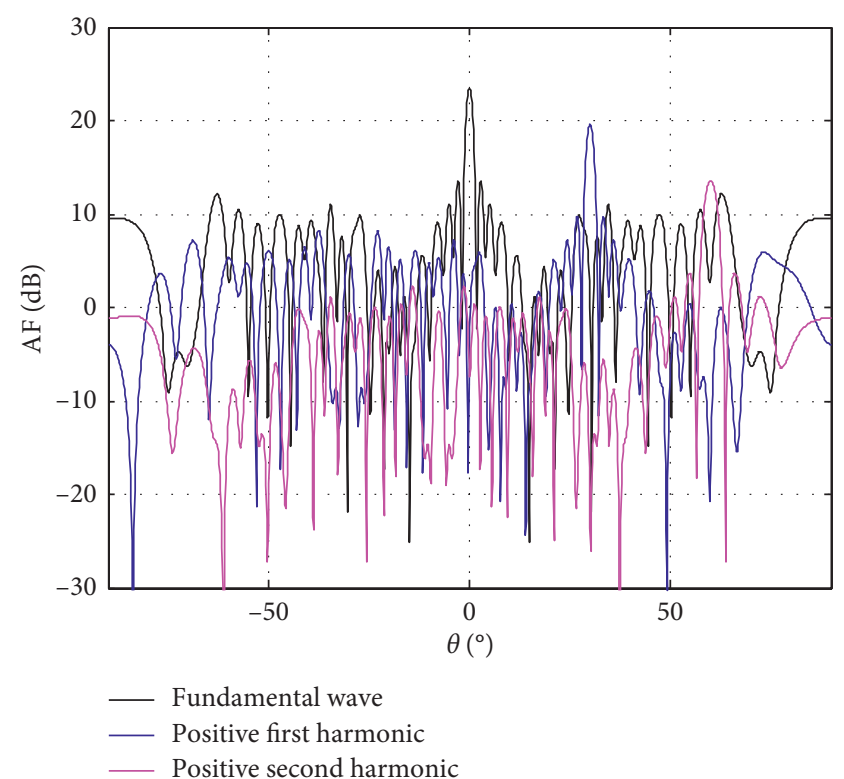

Figure 4: Array factor pattern fundamental wave and first two positive harmonics of subarray 1.

pattern performance becomes closer, and the fundamental wave and the two subarrays are positive. Besides, the PSL of the positive first harmonic of the fundamental and the two subarrays are further optimized. The PSL of the fundamental wave is downregulated to $-13.78 \mathrm{~dB}$, while the PSLs of the positive first harmonic of the subarray 1 and 2 to $-12.89 \mathrm{~dB}$ and $-13.65 \mathrm{~dB}$, respectively. Besides, the $-3 \mathrm{~dB}$ bandwidths of the preoptimized fundamental wave, the positive first harmonic of subarray 1, and subarray 2 are 1.66, 1.68, and 1.93 degrees, respectively. Though the PSL of each beam is reduced through optimization, the bandwidth variations are negligibly small. In particular, they are $1.96,2.04$, and 2.22 degrees, only upregulated by $0.30,0.36$, and 0.29 degrees, respectively. Accordingly, no additional constraint bandwidth is imposed in this study. It can be concluded that the antenna pattern performance is considerably improved by 
TABLE 1: The parameters to improve the DE algorithm.

\begin{tabular}{lcc}
\hline Parameter & Significance & Value \\
\hline $\mathrm{NP}$ & Number of initial population & 50 \\
$x_{\max }$ & Maximum velocity & 0.5 \\
$x_{\min }$ & Minimum velocity & 0 \\
$F(1)$ & Initial value of $F$ & 0.4 \\
$G_{\mathrm{m}}$ & Maximum number of iteration & 200 \\
$\mathrm{CR}_{\mathrm{l}}$ & Minimum number of adaptive crossover & 0.1 \\
$\mathrm{CR}_{\mathrm{h}}$ & operator \\
& Maximum number of adaptive crossover & 0.9 \\
\hline
\end{tabular}

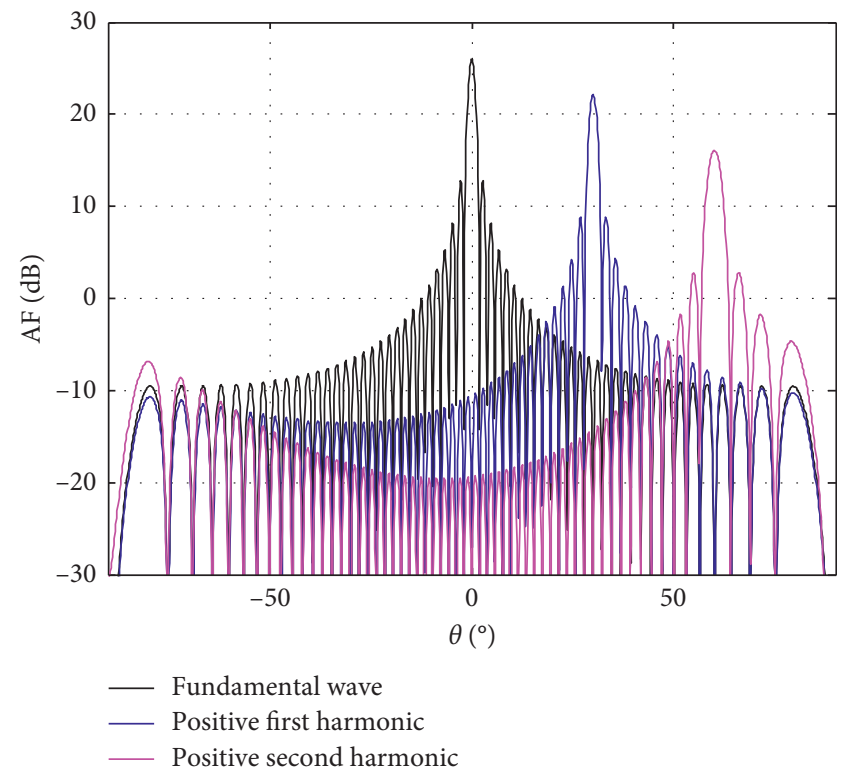

Figure 5: Array factor pattern fundamental wave and first two positive harmonics of fully populated array.

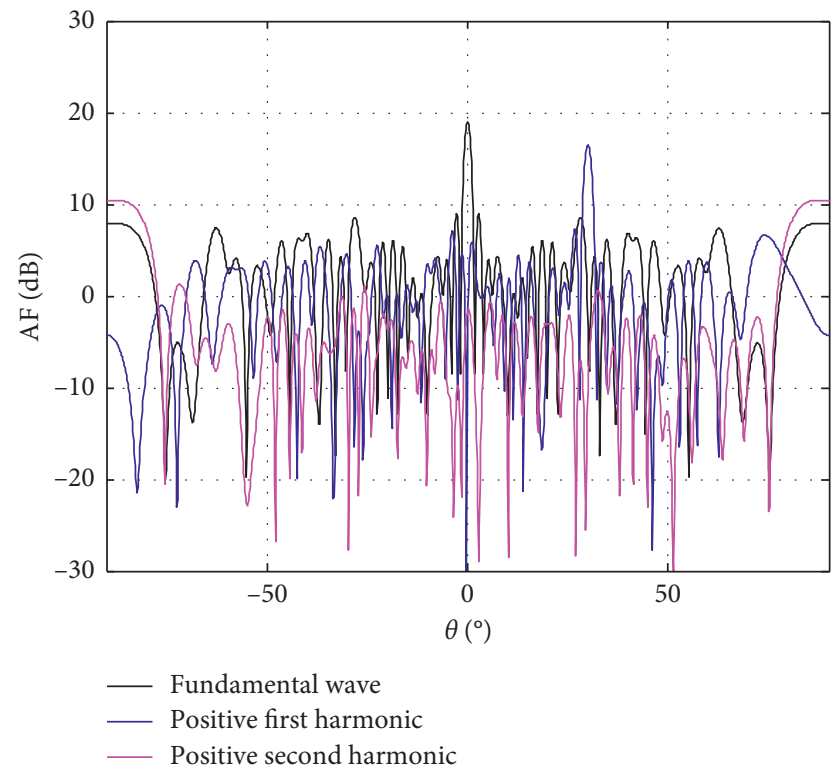

FIgURE 6: Optimized array factor pattern fundamental wave and first two positive harmonics of subarray 1 .
TABLE 2: The gain of different algorithm.

\begin{tabular}{lcccc}
\hline & $\begin{array}{c}\text { Element } \\
\text { number }\end{array}$ & $\begin{array}{c}\mathrm{Gain}_{1} \\
(\mathrm{~dB})\end{array}$ & $\begin{array}{c}\mathrm{Gain}_{2} \\
(\mathrm{~dB})\end{array}$ & $\begin{array}{c}\Delta \text { Gain } \\
(\mathrm{dB})\end{array}$ \\
\hline $\begin{array}{l}\text { Subarray 1 } \\
\begin{array}{l}\text { Fully populated } \\
\text { array }\end{array}\end{array}$ & 29 & 19.60 & 13.57 & 6.03 \\
$\begin{array}{l}\text { Improved DE } \\
\text { subarray 1 }\end{array}$ & 60 & 22.09 & 15.73 & 6.36 \\
\hline
\end{tabular}

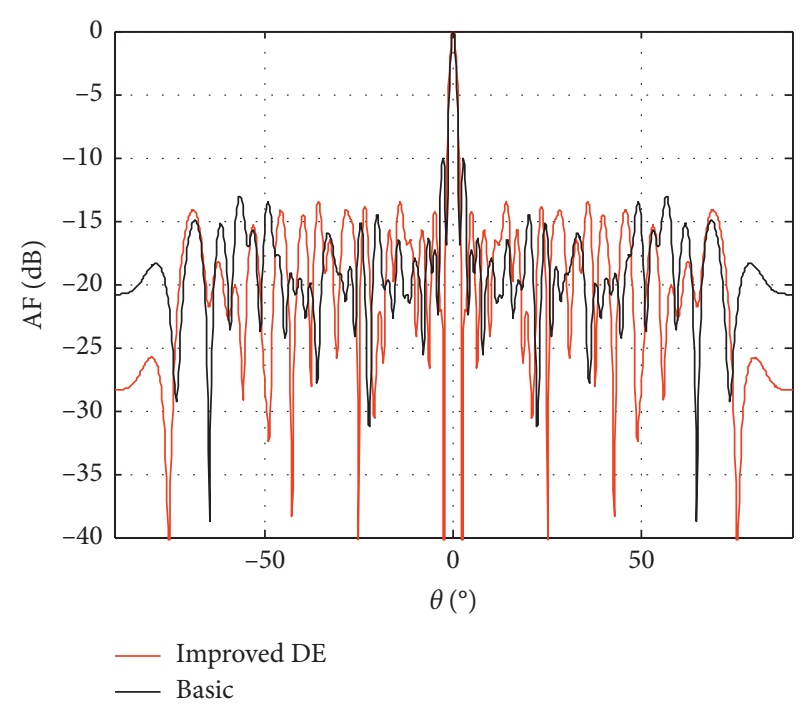

FIgURE 7: Fundamental patterns obtained by optimized DE and basic algorithm.

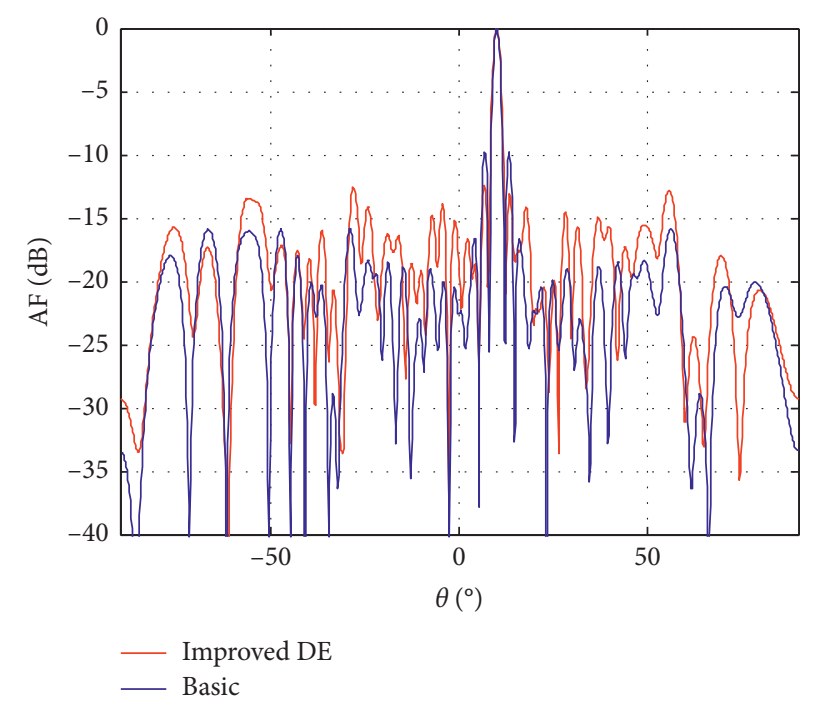

FIGURE 8: The positive first harmonic patterns of subarray $1 \mathrm{ob}-$ tained by optimized DE and basic algorithm.

applying the algorithm proposed in this study and using the duration of the switch openings of the two subarrays as optimization variables.

The iterative curve of the control sequences for the two subarrays optimized by the proposed algorithm is plotted in Figure 10. It can be observed that the optimized update variant mutation operator and the scaling factor of the 


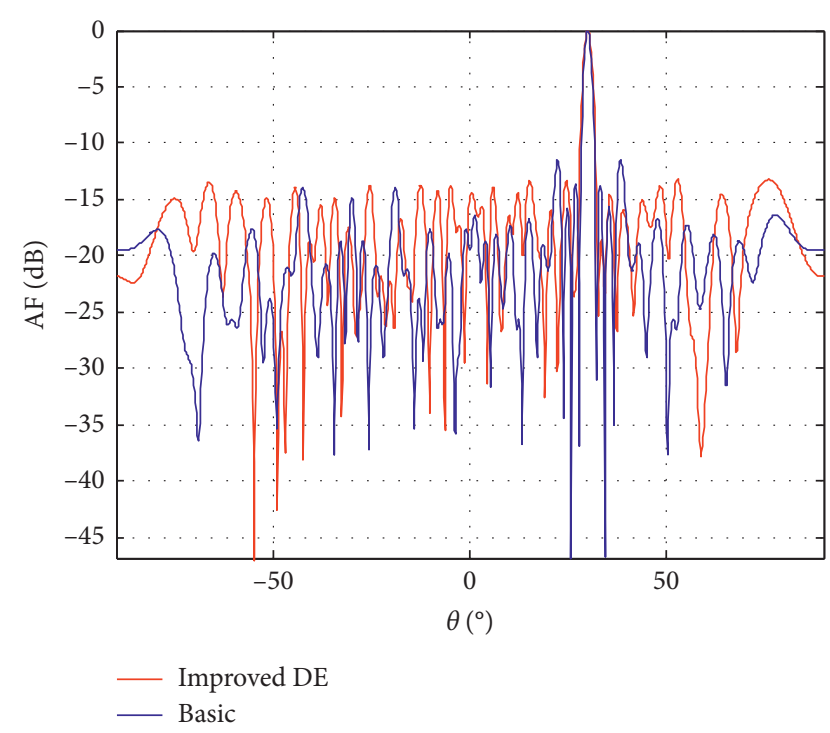

FIGURE 9: The positive first harmonic patterns of subarray $2 \mathrm{ob}-$ tained by optimized DE and basic algorithm.

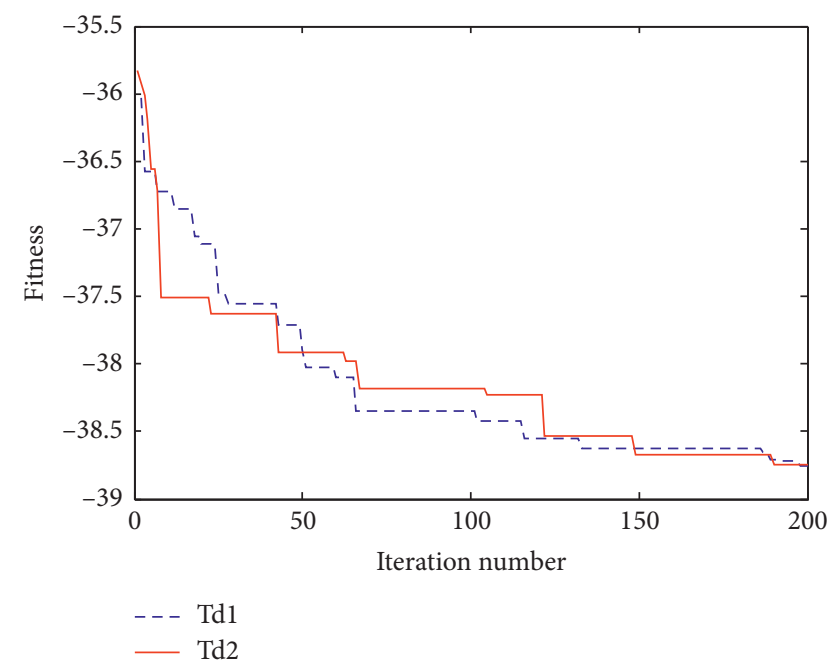

FIGURE 10: Iterative curve of subarray 1 and subarray 2 optimized variables.

sinusoidal iterative chaotic system can enhance the diversity of the population. Thus, the objective function value is very useful.

The location of the subarray element after optimization by the algorithm is as follows:

Subarray 1: 1001101010110101001001010101010111010 10110100110110101010010

Subarray 2: 0110010101001010110110101010101000101 01001011001001010101101

4.2. Simulation Experiment 2. To further verify the feasibility of the proposed algorithm, the proposed algorithm is compared with the iterative FFT algorithm. The algorithm in this study can yield more beams simultaneously

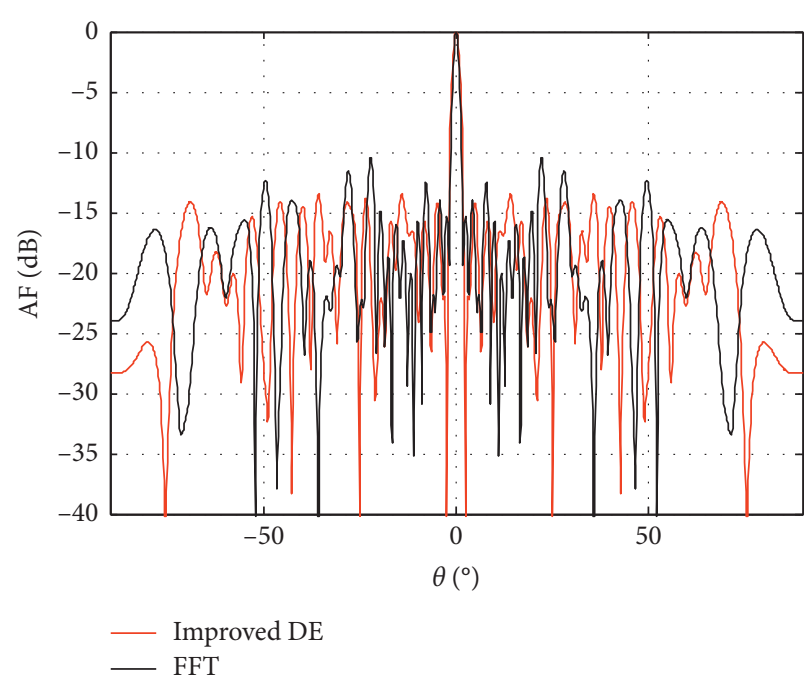

FIGURE 11: Fundamental patterns obtained by optimized DE and FFT algorithm.

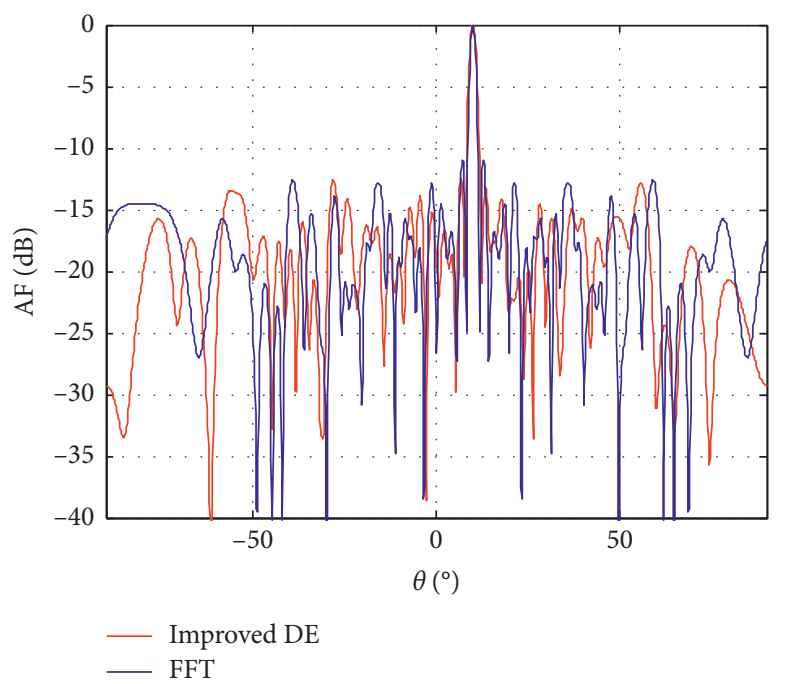

Figure 12: The positive first harmonic patterns of subarray 1 obtained by optimized DE and FFT algorithm.

than the iterative FFT. The fundamental wave to point was set to $0^{\circ}$, while the positive first harmonic of subarray 1 points to $10^{\circ}$, and the positive first harmonic of subarray 2 points to $30^{\circ}$. The beam performance of the two methods is compared, and the simulation results are shown in Figures 11-13.

Compared with the iterative FFT algorithm, the proposed algorithm exhibits the following two advantages. First, in the case of a certain number of array elements, the algorithm can form more beams while the beam directions are flexible. Second, the PSL of the formed multibeam is lower, and the pattern performance is superior to the iterative FFT performance. The results of simulations 11,12 , and 13 suggest that the PSL of the beam pointing to $0^{\circ}$ formed by the iterative FFT algorithm is $-10.47 \mathrm{~dB}$, while it is $-10.96 \mathrm{~dB}$ and $-10.49 \mathrm{~dB}$ for $10^{\circ}$ and $30^{\circ}$, respectively. However, beams 


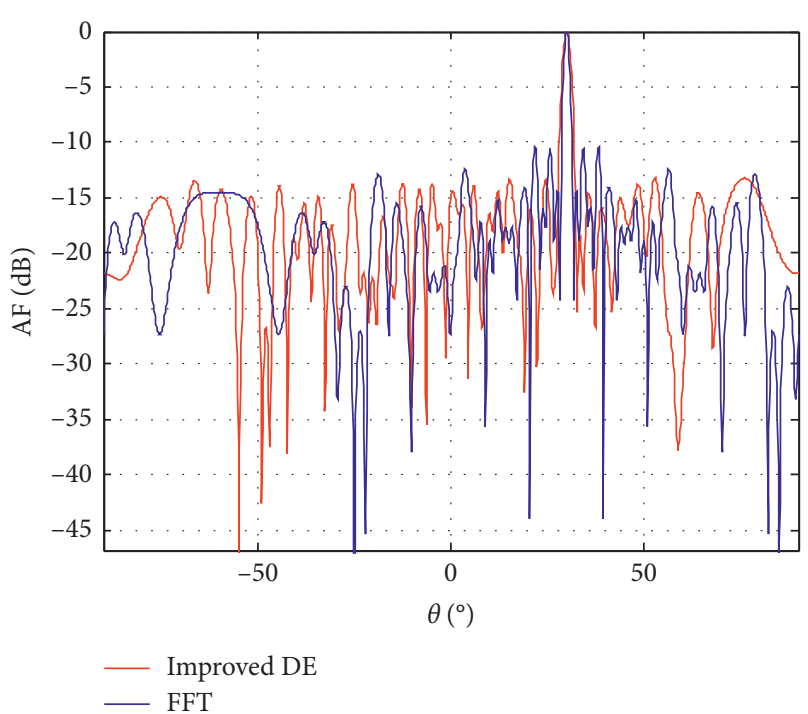

FIgURE 13: The positive first harmonic patterns of subarray 2 obtained by optimized DE and FFT algorithm.

formed by the algorithm in this study are better. The PSL of the fundamental wave formed is $-13.48 \mathrm{~dB}$, while the PSL of the positive first harmonic of subarrays 1 and 2 are $-12.52 \mathrm{~dB}$. These values are $3.01 \mathrm{~dB}, 1.56 \mathrm{~dB}$, and $2.96 \mathrm{~dB}$ lower than the PSL of the beam corresponding to the iterative FFT algorithm, respectively. The above simulations reveal that the optimized $\mathrm{DE}$ algorithm has a better direction in the graph and has a faster convergence speed. The algorithm can adaptively adjust the mutation weight of the mutation operator according to the current state of the individual population, avoiding the early maturity attributed to the premature falling into the local optimum, and it exhibits better beam performance than other conventional methods.

\section{Conclusion}

In this study, TMLA technology is employed to design the shared aperture multibeam forming method for the first time. More beams are formed after the shared apertures are sparsely interleaved to form two subarrays. The optimized DE algorithm, introducing the scaling factor of the sinusoidal iterative chaotic system and the adaptive cross-probability factor, is presented to optimize the control sequences of the two subarrays. The PSLs of the fundamental wave and the harmonics, formed by the two subarrays optimized by optimized DE, were revealed to be lower than those optimized by other algorithms. Simulation results confirm the feasibility of the proposed algorithm.

The TMLA technology can achieve the beam control in the antenna array. In the meantime, it not only has the characteristics of simple system structure and low cost but also has a very practical significance for realizing miniaturization of the phased array system. Thus, TMLA will be further applied to the multibeam forming of the conformal array.

\section{Data Availability}

We note that there are no data sharing issues since all of the numerical information is produced by solving the equations of the proposed algorithm, which are realized by the Matlab software in the paper. The simulation data and programs used to support the findings of this study are available from the corresponding author upon request.

\section{Conflicts of Interest}

The authors declare that there are no conflicts of interest regarding the publication of this paper.

\section{Acknowledgments}

This work was supported by the National Natural Science Fund (nos. 61871203 and 61671239) and Postgraduate Research \& Practice Innovation Program of Jiangsu Province (KYCX19_1687) in China.

\section{References}

[1] X. Yang, H. Xing, and X. Ji, "Sound source omnidirectional positioning calibration method based on microphone observation angle," Complexity, vol. 2018, Article ID 2317853, 15 pages, 2018.

[2] D.-X. Wang, M.-S. Jiang, F.-L. Niu, Y.-D. Cao, and C.-X. Zhou, "Speech enhancement control design algorithm for dual-microphone systems using $\beta$-NMF in a complex environment," Complexity, vol. 2018, Article ID 6153451, 13 pages, 2018.

[3] S. Gong, W. Long, H. Huang, D. Ben, and M. Pan, "Polyphase orthogonal sequences design for opportunistic array radar via HGA," Journal of Systems Engineering and Electronics, vol. 24, no. 1, pp. 60-67, 2013.

[4] L. Li, "Research on the shared aperture multifunction wideband array antenna," Journal of Xidian University, vol. 43, no. 4, pp. 147-153, 2016.

[5] S. Quan, W. Qian, J. Guq, and V. Zhang, "Radar-communication integration: an overview," in Proceedings of the IEEE 7th International Conference on Advanced Infocomm Technology (ICAIT), pp. 98-103, Fuzhou, China, November 2014.

[6] H. Zeng, Y. Wang, and L. Liu, "An adaptive iterative broadband DBF algorithm," Journal of Astronautics, vol. 35, no. 11, pp. 1306-1311, 2014.

[7] C. I. Coman, I. E. Lager, and L. P. Ligthart, "A deterministic solution to the problem of interleaving multiple sparse array antennas," in Proceedings of the European Radar Conference, Piscataway, NJ, USA, October 2005.

[8] Y. Qin, S. Gao, A. Sambell, M. Elsdon, and E. Korolkiewicz, "Design of a broadband square-ring-slot coupled patch antenna," Microwave and Optical Technology Letters, vol. 47, no. 5, pp. 454-457, 2005.

[9] M. Simeoni, I. E. Lager, C. I. Coman, and A. G. Roederer, "Implementation of polarization agility in planar phasedarray antennas by means of interleaved subarrays," Radio Science, vol. 44, no. 5, pp. 1-12, 2009.

[10] J. Hu, B. Wang, and L. Li, "Synthesis optimization of sharedaperture interleaved arrays," Electronics Optics \& Control, vol. 20, no. 8, pp. 33-36, 2013.

[11] L. Li, B. Wang, C. Xia et al., "Design of array with multiple interleaved subarrays based on subarray excitation energy- 
matching," Journal of Beijing University of Aeronautics and Astronautics, vol. 42, no. 11, pp. 2395-2402, 2016.

[12] M. A. Panduro, A. Reyna, and J. Camacho, "Design of scannable linear arrays with amplitude and phase optimisation for maximum side lobe level reduction," International Journal of Electronics, vol. 96, no. 3, pp. 323-329, 2009.

[13] M. A. Panduro, C. A. Brizuela, D. Covarrubias, and C. Lopez, "A trade-off curve computation for linear antenna arrays using an evolutionary multi-objective approach," Soft Computing, vol. 10, no. 2, pp. 125-131, 2006.

[14] L. Li, B. Wang, and C. Xia, "Thinned and interleaved planar array antenna based on iterative FFT techniques," Chinese Journal of Radio Science, vol. 31, no. 2, 2016.

[15] L. Li, B. Wang, and C. Xia, "Interleaved thinned linear arrays based on modified iterative FFT technique," Journal of Electronics \& Information Technology, vol. 38, no. 4, pp. 970-977, 2016.

[16] X. Liu, Z. Zhang, and X. Fei, "Multi-aperture beamforming with shared aperture based on simulated annealing algorithm," Electronics Optics \& Control, vol. 25, no. 11, pp. 57-61, 2018.

[17] X. Fei, Z. Zhang, Y. Tian, and X. Liu, "Shared aperture pattern synthesis based on wind driven optimization," Electronics Optics \& Control, vol. 25, no. 6, pp. 52-55+82, 2018.

[18] Y. Ma, S. Yang, Y. Chen, S.-W. Qu, and J. Hu, "Pattern synthesis of 4-D irregular antenna arrays based on maximumentropy model," IEEE Transactions on Antennas and Propagation, vol. 67, no. 5, pp. 3048-3057, 2019.

[19] P. Rocca, L. Poli, G. Oliveri, and A. Massa, "Synthesis of subarrayed time modulated linear arrays through a multi-stage approach," IEEE Transactions on Antennas and Propagation, vol. 59, no. 9, pp. 3246-3254, 2011.

[20] R. Maneiro-Catoira, J. C. Bregains, J. A. Garcia-Naya, L. Castedo, P. Rocca, and L. Poli, "Performance analysis of time-modulated arrays for the angle diversity reception of digital linear modulated signals," IEEE Journal of Selected Topics in Signal Processing, vol. 11, no. 2, pp. 247-258, 2017.

[21] Y. Tong and A. Tennant, "A two-channel time modulated linear array with adaptive beamforming," IEEE Transactions on Antennas and Propagation, vol. 60, no. 1, pp. 141-147, 2012.

[22] P. Rocca, Q. Zhu, E. T. Bekele, S. Yang, and A. Massa, "4-D arrays as enabling technology for cognitive radio systems," IEEE Transactions on Antennas and Propagation, vol. 62, no. 3, pp. 1102-1116, 2014.

[23] Y. Feng, S. Yang, Y. Chen, S. Qu, and J. Hu, "Convex optimization of pencil beams through large-scale four-dimensional antenna arrays," IEEE Transactions on Antennas \& Propagation, vol. 66, no. 7, pp. 3453-3462, 2018.

[24] A. Reyna, M. A. Panduro, G. Romero, J. Villanueva, F. F. Rivera, and A. L. Méndez, "Two-dimensional timedomain antenna arrays for optimum steerable energy pattern with low side lobes," International Journal of Antennas and Propagation, vol. 2014, Article ID 869192, 12 pages, 2014.

[25] Z. Medina, A. Reyna, M. A. Panduro, and O. Elizarraras, "Dual-band performance evaluation of time-modulated circular geometry array with microstrip-fed slot antennas," IEEE Access, vol. 7, pp. 28625-28634, 2019.

[26] A. Reyna and M. A. Panduro, "Synthesis of timed antenna arrays for flat-top energy patterns," IETE Technical Review, vol. 34, no. 1, pp. 58-65, 2016.

[27] A. Reyna and M. A. Panduro, "Timed Vivaldi arrays for steerable energy pattern with low side lobes," Journal of Electromagnetic Waves and Applications, vol. 29, no. 10, pp. 1375-1383, 2015.
[28] A. Reyna, G. Romero, A. L. Méndez, and M. A. Panduro, "Timed arrays of spiral antennas for circularly polarised UWB scanned patterns with low side lobes," IET Microwaves, Antennas \& Propagation, vol. 10, no. 5, pp. 587-593, 2016.

[29] Z. Huang and Y. Chen, "An improved differential evolution algorithm based on adaptive parameter," Journal of Control Science and Engineering, vol. 2013, Article ID 462706, 5 pages, 2013.

[30] X. Wang and S. Zhao, "Differential evolution algorithm with self-adaptive population resizing mechanism," Mathematical Problems in Engineering, vol. 2013, Article ID 419372, 14 pages, 2013.

[31] A. Reyna, L. I. Balderas, and M. A. Panduro, "Time-modulated antenna arrays for circularly polarized shaped beam patterns," IEEE Antennas and Wireless Propagation Letters, vol. 16, pp. 1537-1540, 2017.

[32] G. Bogdan, Y. Yashchyshyn, and M. Jarzynka, "Time-modulated antenna array with lossless switching network," IEEE Antennas and Wireless Propagation Letters, vol. 15, pp. 1827-1830, 2016.

[33] K. Price, R. M. Storn, and J. A. Lampinen, Differential Evolution: A Practical Approach to Global Optimization, Springer Science \& Business Media, Berlin, Germany, 2006.

[34] G. Li, S. Yang, G. Wang, and Z. Nie, "Mutual-coupling compensation in time-modulated antenna arrays for flat-top pattern synthesis," Electromagnetics, vol. 29, no. 6, pp. 499$507,2009$.

[35] G. Quaranta, G. C. Marano, R. Greco, and G. Monti, "Parametric identification of seismic isolators using differential evolution and particle swarm optimization," Applied Soft Computing Journal, vol. 22, pp. 458-464, 2014.

[36] K. Worden and G. Manson, "On the identification of hysteretic systems-part I: fitness landscapes and evolutionary identification," Mechanical Systems and Signal Processing, vol. 29, pp. 201-212, 2012.

[37] L. Poli, P. Rocca, G. Oliveri, and A. Massa, "Harmonic beamforming in time-modulated linear arrays," IEEE Transactions on Antennas and Propagation, vol. 59, no. 7, pp. 2538-2545, 2011.

[38] G. Li, S. Yang, Y. Chen, and Z.-P. Nie, "A novel electronic beam steering technique in time modulated antenna array," Progress in Electromagnetics Research, vol. 97, pp. 391-405, 2009. 


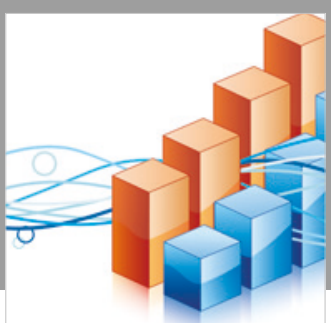

Advances in

Operations Research

\section{-n-m}
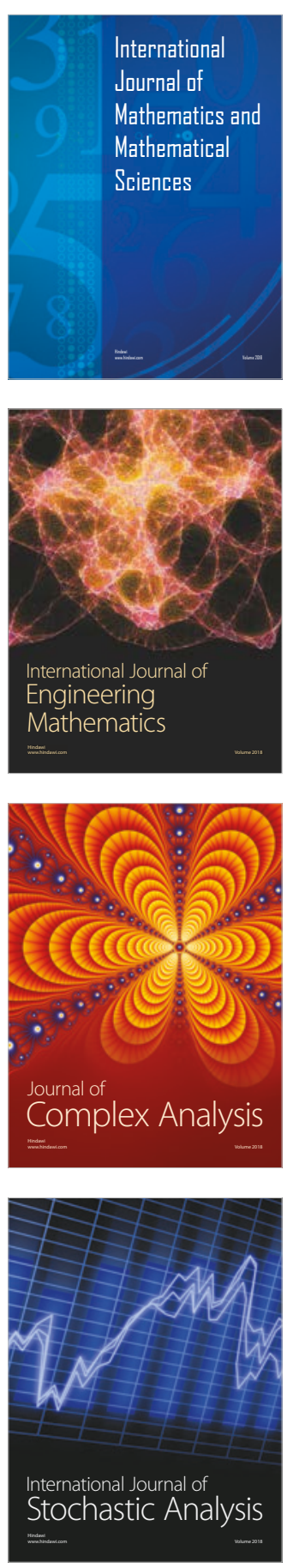
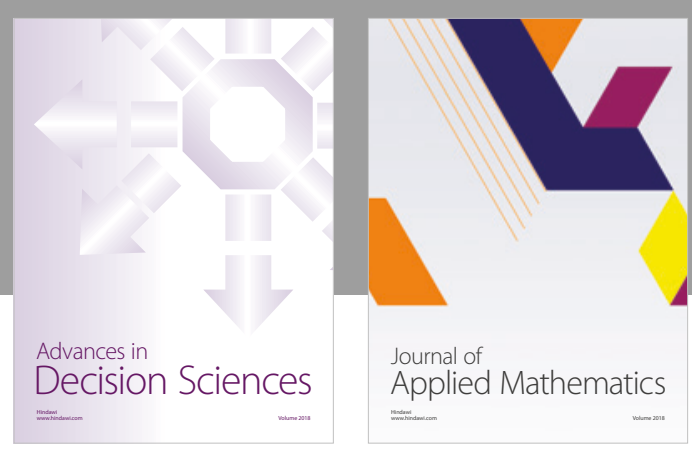

Journal of

Applied Mathematics
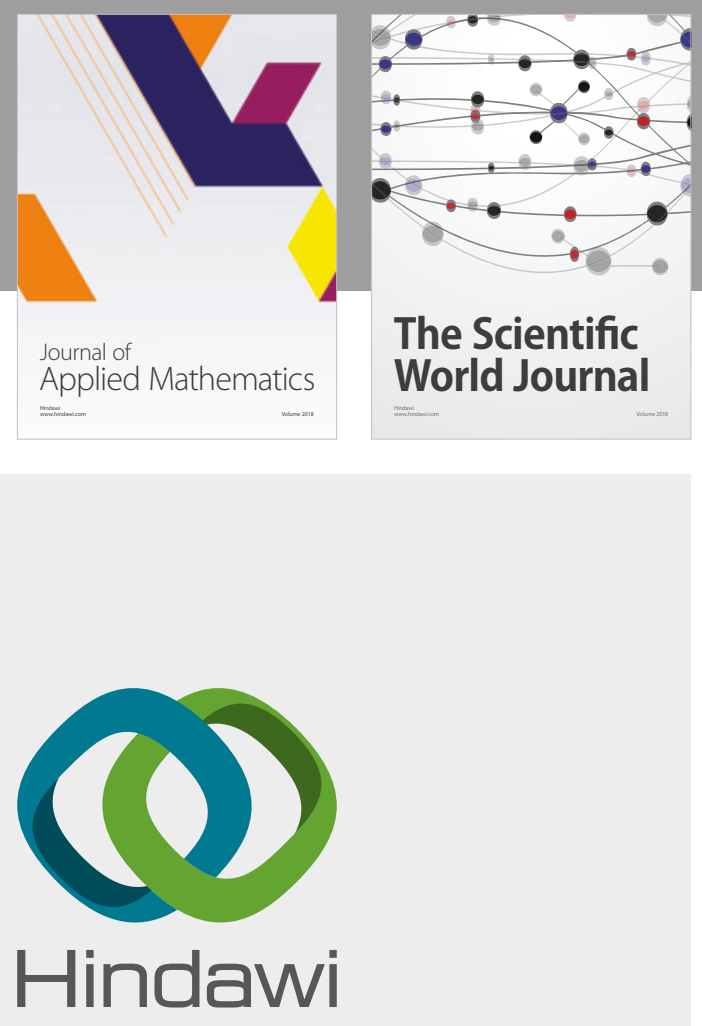

Submit your manuscripts at

www.hindawi.com

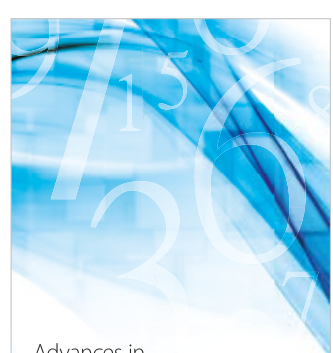

Advances in
Numerical Analysis
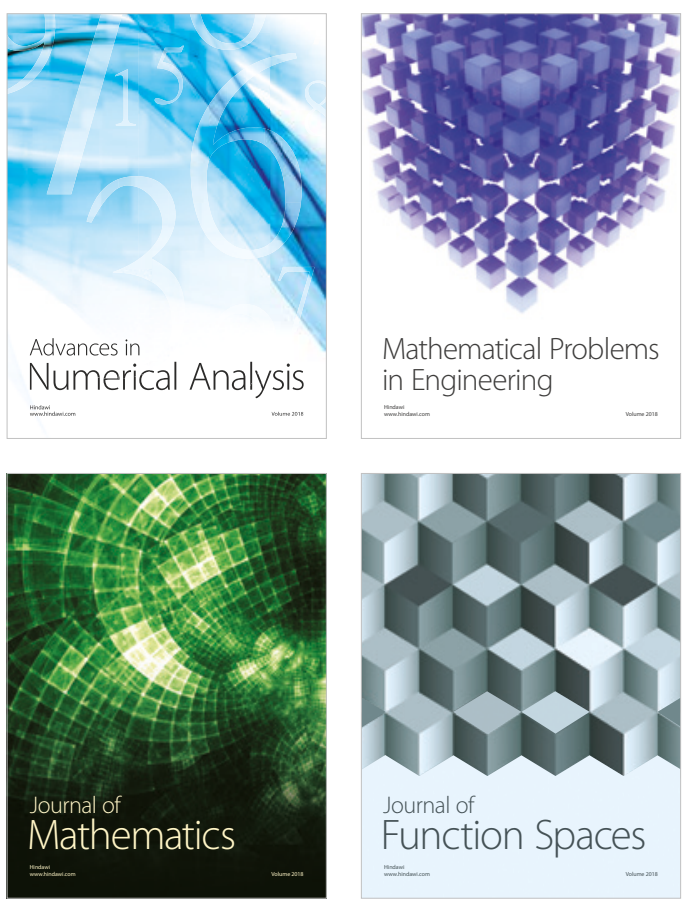

Mathematical Problems in Engineering

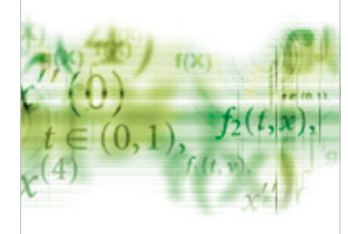

International Journal of

Differential Equations

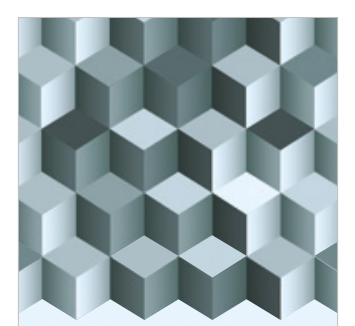

Journal of

Function Spaces
The Scientific

World Journal

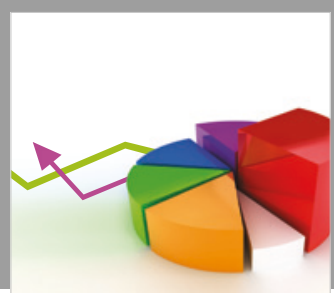

Journal of

Probability and Statistics
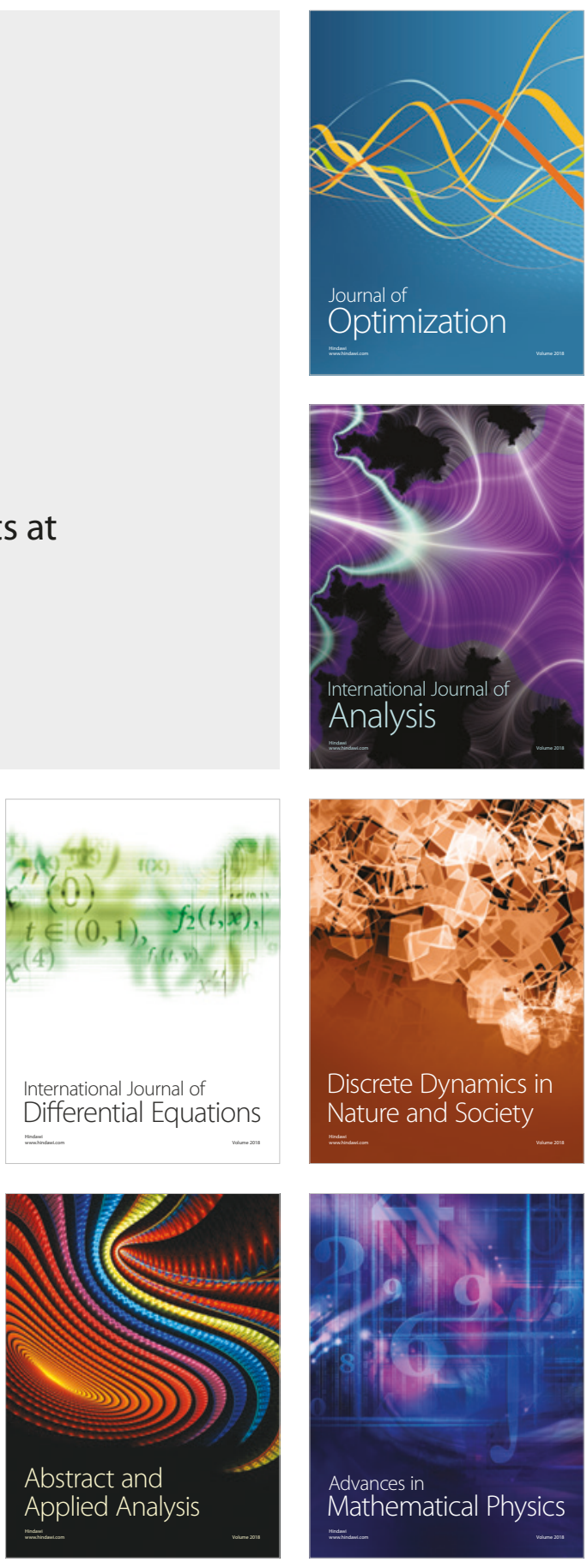